my best to eliminate. A few words will deal with the other possibilities that have been raised. The patient presents symptoms generally of dyspncea, always of fatigue on slight exertion, lassitude, persistent tachycardia without cardiac enlargement, and perhaps a variable number of neurotic manifestations. There is invariably a history of psychic trauma-it may be one acute occasion or a long-continued bombardment by greater or smaller shocks.

These are the cases for exhaustive investigation, for the detection of toxins, for the estimation of buffer-salts, for the consideration of the balance of the ductless glands, for inquiry into the previous psychical history.

It is unsatisfactory to invoke toxæmia, for surely this factor can never be eliminated and must play a part more or less in the production of any morbid state. The system is probably in a condition of auto-intoxication whenever it falls a victim even to psychic disturbances. In some of these cases the antecedent history clearly exhibits the presence of some infection-e.g., influenza, dysentery, or toxic state, such as constipation or some other cause of autointoxication. In other cases it does not.

As regards the ductless glands, I do not see how it is possible to deny or to prove their influence in this condition. What is the effect of their secretions upon the emotions? What is the influence of the emotions upon their secretions? Suppose the psychic disturbance does lead to some alteration in the glands, to the production, for example, of hyperthyroidism. Are we to suppose that such alteration is the principal result of the shock and that treatment directed to the gland will relieve symptoms? I personally have never seen the slightest benefit derived from this or, for that matter, any other form of treatment.

There is, it seems to me, a great deal to be said in favour of a purely nervous origin. In the first place, a very large proportion of sufferers are of a distinct type of mentality, men with shallow reservoirs of nervous energy - the neurasthenic soil, in fact. I yield to nobody in my admiration of the spirit which has animated the majority of sufferers from "soldier's heart" and all other forms of war neurasthenia when I describe them in this way. The symptoms correspond to a failure in vaso-motor and cardio-inhibitory control. The obstinate resistance to any form of treatment supports the idea of a nervous origin, a shock to the nerve centres which persists who can say for how long It is a very striking feature that men who sustain a definite somatic injury do not manifest "soldier's heart," nor any other presumably nervous symptoms. In these cases of injury the immunity of the nerve centres to shock may be explained by the dissipation of the shock elsewhere, as the delicate works of a watch are spared in an accident in which the glass is smashed. It may even be explained, without detriment to the hypothesis of neurosis, by the mere circumstance of an injury having occurred satisfying the subconscious with the realisation of something definite, and not presenting to it only the sensation of some vague disturbance, the uncertainty of which prejudices recovery.

Reviewing, with not unnatural pessimism, the persistence of symptoms, one cannot help thinking that only termination of hostilities could cure these sufferers, in whom with the best of intentions there must continue to run a subconscious current of defence-neurosis telling them that recovery means a return to the hell from which they have escaped. Treatment of such cases appears to have the sole effect of perpetuating their neurosis by fixation of the attention, although whether immediate vigorous treatment of them as of purely neurotic origin would yield better results we here have no opportunity of observing, since the cases we see are, so to speak, chronics who are left in statu quo after a great deal of practically every form of therapy that has been recommended. As we see them, at any rate, such cases are of no further use for general service, but given sedentary work they continue on a low level of activity with the tachycardia and other symptoms unrelieved, but, so far as we can see, executing very light duties with comparative cheerfulness and apparently without any ill effects.

ST. Austell (CoRnWall) Board of Guardians AND THE TREATMENT OF ADENOIDS.-At the last meeting of the $\mathrm{St}$. Austell board of guardians it was decided that Dr. A. Shaw should operate upon children requiring the removal of adenoids at a fee of 1 guinea per patient.

\section{IMPORTANT PRINCIPLES IN THE DRAINAGE AND TREATMENT OF WOUNDS.}

\author{
By WILLIAM PEARSON, F.R.C.S. IREL, \\ MAJOR, R.A.M.C. (TEMP.).
}

With Remarks by P. J. FreYer, C.B., Lieutenant-Colonel, I.M.S. (R.), Consulting Surgeon, Eastern Command.

AN experience of more than two years of war surgery, with considerable study of its literature, has convinced me that no general agreement in the principles of wound treatment has yet been reached amongst surgeons. My time has been spent first in France and subsequently in Fingland, and several thousands of cases have passed through my care, so that I feel I can speak with some authority on the behaviour and progress of various types of wounds in both their early and later stages. This dual experience is, I believe, of value in helping one to form a clear judgment on the merits of different lines of treatment, because it has appeared not infrequently that the views of workers in France and of those in this country are at variance; and it has been suggested that these differences are due to the different conditions under which the work is performed, or rather to the different stages in the life-history of the wounds at which treatment and observations are carried out, so that both may be right, and what is sauce for the goose is not alvays sauce for the gander. I do not believe that this is a satisfactory explanation, as the general lines on which I was working when I left France two years ago are, with slight modifications, those that I still employ with excellent results. The modifications have not been changes of principles, but rather a further advance on the same lines.

Naturally a very large amount of the work and literature on wounds has been devoted to the questions of infection and sepsis and their treatment; and it is not uncommon to hear it said that our ideas on these subjects were previously all wrong, and that we have had to change both ideas and methods entirely. Infection and sepsis are no new things ; the differences between them as seen in war wounds and in civil practice are differences of degree and character only, so that if the previous statement is correct it follows that heretofore surgeons have been working on altogether wrong principles in dealing with them. But this is manifestly incorrect, as evidenced by our enormous strides in dealing with intra-abdominal infections, to take only one example. " Ubi pus, ibi evacua" is a surgical maxim which is as generally accepted as it is old, and all surgeons are agreed on the necessity for drainage in dealing with sepsis.

\section{Claims as to Value of Antiseptics and Concentrated Saline.}

Concerned with this question of drainage are two schools -I had almost said factions-which hold different views as to how drainage may best be aided in subduing infection. The members of one, which may be termed the "antiseptic school," pin their faith to the nse of autiseptic solutions by which they believe they can rapidly diminish or destroy the organisms in situ, and thus subdue the sepsis and even render the wound sterile. The doctrine of this school amounts to an admission that by drainage alone they are unable to effect satisfactory results - in other words, as I shall indicate presently, that their drainage is faulty or inadequate. The weakness of their position is indicated by the truly astonishing number and variety of antiseptics which have been advocated for this purpose in the medical press of the past two years. From amongst them the hypochlorite group. exemplified by "eusol," may be taken as being the most fashionable at the present time.

The other school, ably led by Colonel Sir AJmroth Wright, may be called the "concentrated saline school." It maintains that by antiseptics it is impossible to destroy all organisms in the wound once infection is established. short of destroying the tissues themselves, and that infection and sepsis are best combated by promoting free secretion into and discharge from the wound, making use of a concentrated saline solution for this purpose. Whether this acts by osmosis or in some other way is really immaterial to the issue. provided it is effective and necessary. The solution advocated by Sir Almroth Wright is 
one of sodium chloride 5 to 10 per sent., and it has been enthusiastically supported by $m \approx n y$ surgeons both in this form and in its modification known as the "salted pack," which consists of a gauze pack in which are incorporated soloids of sodium chloride. While fully appreciating the value of Sir Almroth Wright's work, and recognising that through iti he has been demonstrating and emphasising the importance of drainage, the lessons to be learnt by practical suryeons have, I believe. become somewhat obscured by the undue prominence given to the rôle of the concentrated stine, both in his writings and in those of other supporters. Indeed, that this is so was made manifest to me by the remark of a distinguished surgeon, when discussing with me the "salted pack" and advocating its merits a year ago. He said: "It is extraordinary the way it works; it seems to have a remarkable instinct in causing a profuse discharge in the very cases in which it is necessary, and causing very little in others where it is not required." Assuredly, the "remarkable instinct," prompted by the organisms, was exercised by Nature and not by the salt in the pack.

Unfortunately the brilliant advncacy of "concentrated saline," and more recently of "eusol," has possibly done harm. I am convinced that through it, in the minds of many, the main issue has become obscured, and that the partmount importance of good mechanical drainage has been lost sight of. It is, even still, not an uncommon thing to receive a patient, whose wound is in a bad state of sepsis and most inadequately drained, adorned with a label on which one reads, "washed out with concentrated saline twice daily"; " irrigated with Wright's saline every four hours"; " treated with salt packs" ; or, " treated with eusol-wounds now beautifully clean." I quote from actual cases received under mo owu care ; the latter case in particular I remember for its cheery optimism, as it was what I would describe as "full of sepsis." I am fully aware of the magnificent surgical work that is being done in France, of the very difficult conditions under which it is often performed, and of the fact that these cases had probably not been dressed for 24 hours when we received them. I only instance them to show that in the minds of some, at least. the value of good mechanical drainage is not fully appreciated, and the use of concentrated saline or the latest fashionable antiseptic is given the seat of honour.

\section{Importance of Free Mechanical Drainage.}

Two months' work in France at a base hospital sufficed to convince me that the use, in a wound, of the antiseptics which were then in vogue was valueless in subduing infection at the time when this was the all-important indication for treatment. I became convinced that the secret of success lay in free mechanical drainage; nor have other measures since advocated, and which $I$ have given a fair trial, caused me to change my opinion.

It is, of conrse, universally agreed that sepsis cannot be dealt with arequatelv either by antiseptics or concentrated saline if unsupported by drainage; but it is not. I think, generally recognised that wound infection can be thoroughly and efficiently controlled by mechanical drainage without the use of either of these aids in the wound itself. And yet $I$ am convinced that this is so, and that the results are equally prompt and satisfactory. Of such paramount importance is $\mathrm{g}$ rod free mechanical drainage, that when this has been properly established it is a matter of indifference whether we employ antiseptic solutions or concentrated saline as an aid, and the value of either is so subsidiary as to be negligible. I will n't assert, as some have done, that, they are injurious-I have formed no definite conclusions on this point-but I do say that they are unnecessary. The greatier my experience in wound treatment the more I am convinced of the truth of this, both frnm my own personal observations and from my knowled ge of the work (sometimes in an nponsite sense) of others Mechanical drainage is the essential faotor in the successful treatment of infection, and success will be directly proportional to the menhanical effi iency of the drainage. The question which every surgeon should ask himself, when faced with a case which is not progressing satisfactorily, is. "What is wrong with the drainage spstem in this wound?" Almost invariably the answer will supply the key to successful treatment, if we exclude, of enurse, cases of severe systemic infection. gas gangrene, and so on, which fortunately form only a small proportion of our cases. Even these will become greatly reduced in number by the early establishment of adequate drainage.

Unfortunately we meet here with the difficulty of the personal equation in the surgeon: what one may regard as adequate another may think quite insufficient. But it may help somewhat to state that what is sufficient drainage in the common infections of sivil prastioe is usually totally inadequate when dealing with a nound infection of similar magnitude, and that it is impossible to over-drain. Indeed, as a general rule, the freer the incisions the more quickly and satisfactorily is the wound healed, a fact which is not apparent at first sight.

Numerous cases might be quoted where, in spite of weeks of treatment, progress was being retarded or the patient going steadily to the bad from want of mechanically efficient drainage; but it will be more to the purpose to point out the more common faults in drainage as $I$ have observed them. They are: 1. Insufficiently free incisions. 2. Drainage against gravity, without counter-openings, especially in thigh, arm, and shoulder cases. 3. Too small, improperly adjusted, or improperly used drainage-tubes. 4 Mechanical obstructions within the wound, such as small or valvular openings from " pockets," hernial protrusions of lacerated tissue (especially muscle), foreign bodies, bone fragments and débris, tight gauze packs. 5. Obstruction external to the wound, such as tight bandages, pressure from splints, bedding, \&c.

In the vast majority of cases the complications and sequelæ of sepsis may be avoided by the institution of timely drainage on sound mechanical principles; and their occurrence should usually be regarded, not as "bad luck," but as an indication that in some way or another the drainage system has been at fault.

\section{Mechanical Principles Involved in Drainage.}

Recognising the paramount importance of drainage, and remembering that from an operative point of view it is merely a mechanical problem, our efforts to render it efficient must be directed in accordance with simple physical laws. Though these are well known, I wish to consider them briefly with special reference to their practical applica. tion to projectile wounds. The chief factors governing the efficacy of drainage are then: (1) Absence or reduction of resistance to outflow; (2) gravity; (3) capillary action. The first two of these are closely connected.

1. The freer the exit the less is the resistance offered to the escape of discharges from the wound. This shows the necessity for free incisions in dealing with infection, particularly in deep wounds. Our aim shonld be to convert these into cone-shaped openings, the base of the cone being situated at the surface, so that free exit is obtained from all parts of the wound; pockets and tracks should be thoroughly opened up ; " tunnels" should usually be laid open throngh. out unless anatomical considerations forbid; obstructions within the wound shnuld be removed, so that good access and free exit are established throughout. The necessity for extensive surface openings is very mush great ur in the case of infections in projectile wounds than in these of civil praotioe, so that it is evident that there is something more than the mere question of free exit involved. The explanation is to be found, I believe. in the nature of the infecting organisms, which usually contain large numbers of anaerobes in the former.

Free drainage in the wound itself will not avail if the out flow is obstructed externally, and therefore attention shonld always be paid to this matter. Dressings should not be applied tightly, splint pressure must be carefully avoided, and the patient must be disposed in bed in such a way that no pressure is exerted over the wound. These are poipts which are frequently neglected. and it is difficult in the course of a paper to frame rules for every case. Briefly, however, I may point out that wounds in the posterior surfaces of thigh or log should be treated by slinging the limb from a Balkan splint or leg cradle; in back and buttock wounds the patient should be placed on a "spinal bed" or framework with removable canvas "slats," so that pressure against the wound mav be avoided-failing such an apparatos the patient should be kept in the lateral rather than in the dorsal position; wounds of the upper limb may often be conveniently treated by suspension in a Thomas' knee-splint or from one of the more elaborate devices. 
2. The value of gravity and the use of couter-openings as an aid to drainage are not, I think, sufficiently appreciated in practice This is particularly so in cases of wounds on the front of the thigh with, perhaps, a compound fracture of the femur. In these it is not uncommon to find, even after weeks of treatment, that the only exit is an overflow, that the whole wound is a foul cesspool of pus which hav tracked extensively in the limb, and that there is widespread necrosis of the bone and complete absence of union in the fracture. Such a condition can easily be avoided if in the first instance, no matter how free the anterior opening may be, a g yod counter-opening is made into the deepest part of the wound-preferably, in thigh cases, from the posteroexternal aspect of the limb.

In extensive wounds with much laceration of tissue it will frequently be necessary to make several counter-openings to deal with different recesses or " pockets," but the surgeon should have a definite purpose in view for each opening he makes; it must never be made by "rule of thumb" but to suit the exigencies of the particular case. The efficient drainage of each individual wound is a problem in itself which should be governed by definite principles indeed, but the details of which depend on the exact characters of the wound. Often it is a simple matter and can be determined at a mere glance; at other times it becomes one of great complexity and cannot be solved without thorough digital exploration and inspection of the wound on the operaingtable. Even then anatomical considerations may prevent one from following the most efficient mechanical plan, and one has to adopt something less. But what I wish to emphasise again is that no other measures whioh we may omploy will adequately replace a mechanically perfeot system of drainage.

3. Capillary action, though of less importance than free exit and gravity, is also a valuable mechanical aid in promoting drainage; it literally "draws" the discharge. In employing gauze wicks and packs for this purpose it is important to observe the following points :-

(a) The gauze should extend into the various parts of the wound, which should be filled up evenly and with but moderate pressure throughout; it should constitute what I term a ganze " fluff," and not a tight pack.

(b) Dead spaces should not be left within the gauze or between the gauze and the walls of the wound, as they will favour stagnation and decomposition of the discharge and form an excellent culture-ground for bacteria.

(o) In order to maintain capillary action it is necessary to prevent water-logging of the gauze drain. Hence we should allow evaporation to take place, and should never employ a waterproof covering over the dressing during the period of profuse discharge. For this reason, too, external dressings of gauze will be much better than close substances such as lint. The time-honnured boric fomentation is to be vehemently condemned at this stage, as it rapidly becomes merely a pus poultice. but unfortunately its use is still common. Copious coverings of wool and the application of firm. heavy bandages should similarly be avoided except for purposes of transit. The use of large quantities of wool for the purpose of soaking discharge should also be condemned on grounds of economy, as much of the wool now in use is anything but absorbent, and discharges merely track along inside the dressing to find their escape in the bedclothes. If the external ganze dressings become saturated they should be changed immeriately.

$(d)$ It is in the acute virulent stage of infection, when the discharge is usually profuse and serous in character, that capillary action is an effective air to crainage; it is of no value when discharge is thick and purulent. If gauze packs are used then, and if discharge is profuse. they become clogged with secretion in a short time, and acting as foreign bodies merely delay the cleansing of the wound. This does not mean that gauze packs should never be employed in the later stages of wound treatment. They have very important uses then, too, but chiefly not for drainage purposes. Space does not permit of dealing with these uses here.

Incidentally I mav remark that if a wound is thoroughly cleansed of foreign material and dead tissue and efficiently drained on the lines indicated, the change in character of the discharge from thin serous to thick purulent is accompanied by such a diminution in amount that there is no atage in which we find a profuse purulent secretion. The occurrence, and particularly the persistence of the latter, should always lead one to suspect either a foreign body, dead tissue (especially bone), or defective drainage. Indeed, many flesh wounds treated on these lines clean up rapidly within a week, so that it is difficult to say that there has been any purulent stage, and they may readily be closed by secondary suture or strapping with adhesive plaster.

\section{Aids to Mechanical Drainage.}

1. Uses and abuses of drainoge-tubes.-A drainage-tube should be employed where a dead space cannot be avoided by gauze packing, or where dead tissue is present whose complete removal is inadvisable during the early stages of infection (e.g., most compound fractures), so that a prolonged period of free discharge is anticipated. The tube should lead down to this point, and it may often lie conveniently in the midst of the gauze packing. so that it does not come into contact with the walls of the wound. It must, however, act in the direction of gravity, if possiblethrough a counter-opening, if necessary. Through the tabe a gauze wick may be introduced with advantage while the discharge is serous in character. Occasionally it is useful to keep both tube and wick long, so that they project for some distance from the wound and in this way exert an increased syphonage or suction action on the discharge and convey it to a convenient receptacle.

In deep wounds where neither dead space nor necrotic tissue exists, but where the escape of discharge is in an upward direction, a tube should be led through a dependent counter-opening into the deepest part; or the wound may be enlarged in such manner that gravity no longer hinders but will favour drainage. Where neither measure is feasible a tube should be led directly through the wound to its deepest part, and through it discharge should be aspirated as it accumulates.

Tubes are also useful for purposes of irrigation in deeper parts, particularly in comminuted fractures with necrosis, where there is always some tendency to retention and decomposition of discharge between the bony fragments. Here, unless the exit is exceptionally free, it is convenient to employ two tubes-one for injection and the other for return of the fluid. These may lie side by side (the "doubled-barrelled" tube) in the wound down to the fracture, but are often more efficacious when introduced from opposite sides-one through a counter-opening. In this way accumulated stale discharge can be washed away thoroughly at frequent intervals.

Another use for drainage-tubes, apart from treating infection, is in maintaining the patency of openings in the soft tissues when they show a tendency to close too quickly to permit of sound healing in a deeper part. Examples of this in connexion with bone cavities of moderate size, after necrosis has been eradicated, will not infrequently occur and need not be alluded to further.

Care must always be taken to select, trim, and insert a drainage-tube to suit the particular requirements of the case. Within limits, the larger the bore of the tube the better. The tube should be introduced dorn to the area to be drained, but not through it-the importance of this has been previously pointed out by Colonel H. M. W. Gray. ${ }^{2}$ Great care must be exercised to prevent the end-opening of the tube from being occluded by contact with tissue, and a useful method to prevent this is to cut it in a "fish-tail" manner. Superfiuous side openings should never be used, and the practice of employing tabes in which these have been made previously haphazard by the theatre sister is to be condemned. When cutting a side opening there should always be some definite purpose in view, such as the escape of discharge from some more superficial plane or area, and it should be placed accurately at such level and in such direction as will ensure its efficiency. It is common to meet with cases of through-and-through wounds of the thigh. arm, or leg, with considerable laceration of muscles and a severe comminuted fracture in which the only attempt at drainage has been the insertion of one long drainage-tube right through the limb and fracture, with a few lateral openings cut anywhere and lying anyhow up against the walls of the wound. This plan is never efficient, and should pot be adopted.

The danger of pressure necrosis from a drainage-tube must always be remembered. For this reason tuhes must 
never be a " tight fit," either a smaller tube must be nsed, or, more frequently, the opening should be enlarged. It is always advisable during the acute stages of infection to insert gauze between the tube and the surrounding tissues. On no account should a drainage-tube be left in contact with the wall of a blood-vessel; otherwise secondary hæmorrhage is almost a certainty. Contact with nerves, ducts, and other important anatomical structures should also be avoided.

Superfluous drainage-tubes should not be employed, and as soon as a drainage-tube has fulfilled its functions it should be discarded. It may seem unnecessary to say this, and yet there appears to be an idea amongst many that a drainage-tube in a septic wound, even if it is not doing much good, is at any rate not doing any harm. There comes a time in the treatment of every wound when the retention of a drainage-tube is doing harm: a tube is at all times a foreign body, and thus its presence is injurious uniess the advantages derived from it outweigh its disadvantages. Its primary use in infections is to maintain a free exit for discharges until the infection has been subdued sufficiently to enable us to dispense with it. Its presence may retard as well as favour healing. More than once I have seen a tube retained in a long narrow track which for weeks past had been "crying out" to be allowed to close, and the removal of the tube resulted in complete healing in a few days.

2. Exposure to air.-The advantages of loose dressings and their exposure to the air to prevent saturation and stagnation of discharge and to favour drainage by capillary action have been mentioned already ; but exposure of the wound itself is sometimes of special value.

In the early stages of severe infection, when first a wound has been opened up, I have sometimes found it advantageous merely to cover the wound with one or two thin layers of gauze, which are laid evenly on contact with the surface all over, and to use a bed of dressings on to which the discharge runs. This is best suited to widely gaping or open wounds such as the raw ends of septic "chopped-off" amputation stumps, and I know no method by which they clean up and become covered with healthy granulations more quickly. Presumably, in addition to the continuous and thorough removal of all discharges, this method has the advantage of combating anaerobic infection very effectively, and I have termed it the "local open-air treatment." I frequently adopt this method also for a few days in the later stages of treatment as a preliminary to secondary suture and skingrafting, and $I$ believe it is of real value.

3. Irrigation.-Continuous irrigation has been recommended by many, and is undoubtedly a means of effecting a constant removal of waste products. But it is at best in most cases a troublesome method to carry out; it is impracticable when dealing with large numbers of patients, and I do not think it yields better results than good mechanical drainage without its aid. For these reasons I have rarely employed it. Irrigation of the wound should, however, be performed when packs are being changed, as it not only helps to loosen the latter, but also removes any residual discharge and "floating" organisms. It is also useful for washing away discharge at intervals from areas which otherwise cannot be thoroughly drained (e.g., between bone fragments).

4. Use of concentrated solutions of salts. - Sufficient has been said before to indicate that I do not believe in the efficacy of this method. When drainage is carried out on proper mechanical lines it is quite unnecessary, and I cannot satisfy myself that it either increases the discharge or hastens cleansing of the wound. And here I must allude to an admirable paper by Captain J. E. H. Roberts and Captain R. S. S. Statham on "The Salt Pack Treatment of Infected Gunshot Wounds." 2 It is certainly the best paper I have read on the general treatment of wounds, and $I$ can readily credit the excellence they claim for their results. But $I$ think they have been unfortunate in the title of their paper and that they attribute their success rather to the wrong quarter. Their methods of drainage are very similar indeed to those which I have advocated here, and which I have employed now for two years, and $I$ am convinced that their results will be equally good if they abandon the use of the salt in their packs.

2 Brit. Med. Jour., August 26th, 1916.
5. Admunistration of fluids to the patient.-The discharge from a wound which is its natural irrigant is derived from the body fluids. When the discharge is profuse these fluids will rapidly become depleted and the discharge will diminish, and the elimination of waste products and toxins by the kidneys will be reduced unless we maintain the source of supply. Herein lies the value of the free administration of fluids in cases of severe sepsis.

The late Dr. J. B. Murphy, of Chicago, clearly recognised this in advocating proctoclysis in the treatment of general peritonitis, and he suggested that its mode of action in producing a natural irrigation of the peritoneal cavity was by transference of flnid from the lumen of the bowel to the lymphatics, thereby causing a " reversal of the lymph flow in the peritoneum, so that the latter became a secreting and not an absorbing membrane." But it is surely more probable that the "reversal of the lymph flow" is merely the natural protective inflammatory exudate, which is main. tained and increased by sustaining the body fluids to the full. In this way we see the application of the principle of fluid administration to all cases of infection as an aid to drainage. In severe cases with marked toxæmia, where shock or much loss of blood may be anticipated at operation or where very profuse discharge is to be expected afterwards, saline infusions should be given on the operating table and rectal salines should be administered subsequently; in less severe cases rectal salines alone will suffice. In all cases the patient should be allowed unlimited fluids by the mouth, provided the stomach will tolerate them. The free exhibition of fluids will largely prevent or remove the pinched, shrivelled look of severe sepsis; it will give the patient a general feeling of well-being, it will allay the appalling thirst, and it will maintain a free discharge from the wound until the infection is subdued.

\section{Uses of Antiseptios in Wound Treatment.}

Personally, I consider antiseptics valueless as a means of disinfecting a wound once infection has become established, but nevertheless they have their uses in wound treatment. They prevent stale discharges from becoming a breeding ground for bacteria, and so diminish the risk of infection spreading or being conveyed from one wound to another. Consequently they should be employed in the gauze dressings into which the wound drains, and should also be used in irrikating lotions. Soiled dressings should be placed in a receptacle containing some antiseptic solution when they cannot be burnt immediately. It matters little or nothing what antiseptic is employed, provided it is cheap and efficient and has no particular disadvantage ; substances irritating to the skin, such as mercurial salts, should be avoided in the dressings. For irrigation I prefer a lotion containing peroxide of hydrogen, as it possesses special mechanical properties in loosening dressings and packs and in removing débris from the wound.

In the later stages of wound treatment antiseptics may be put to many uses to promote healing. But they are employed then, not for their antiseptic properties, but rather as caustics. astringents, or stimulants to control and direct the growth of granulation tissue in proper directions-a process which may be termed "coaxing the wound."

\section{Vacoines.}

Like most observers, I have not found vaccines helpful in dealing with infections in wounds of war. Perhaps the type of case in which resort to them is still most frequent is one of chronic sinus which has obstinately refused to heal. I believe that in every case of persistent sinus the obstacle to healing is local and mechanical-a sequestrum or focus of caries, a foreign body, drainage against gravity, too small a surface opening, callous rigid walls, or degenerated granulation tissue-and that the correct treatment is to deal with it accordingly. I know of one case in which a sinus persisted for nearly a year following a simple flesh wound in a limb. Eventually the surgeon was about to resort to vaccines, but a skiagram revealed the presence of a bullet in the muscles,

\section{Rest.}

The importance of maintaining the injured part at rest has been previously emphasised by other writers, and therefore needs only passing reference here. 


\section{Removal of Loose Bone Fragments.}

Differences of opinion have been expressed as to the removal of loose fragments in the early stages from a septic comminuted fracture in view of the danger of non-union. I think the general tendency has been towards insufficient removal and that the danger of non-union is over-rated. In these cases we meet with three types of fragments which, according to their size, may conveniently be termed "fragments" proper, "gravel," and "sand." "Gravel" and "sand" should always be removed as thoroughly as possible ; they merely form masses of necrotic débris in which discharge stagnates and organisms flourish. "Fragments" proper should be removed when they retain no connexion with periosteum and soft parts, as they are then avascular and completely necrotic. Fragments which retain their vascular attachments may be left in situ, provided that their presenoe does not impede free meohanical drainage, especially from the medullary cavity. Only one case of non-union has occurred in hundreds so treated.

\section{Complications of Wound Healing.}

1 desire briefly to allude to two of the most important complications which one meets in dealing with wounds :-

1. Deformities from muscular oontractures. - Muscles which are the seat of inflammation are liable to become permanently shortened, and so to lead to crippling deformities, especially in the limbs. Such deformities are usually easy to prevent but difficult to cure. Consequently we should guard against them from the sturt, and maintain the limb in suitable position. Contraction of the hamstrings with permanent flexion of the knee, and of the calf muscles with drop foot, are amongst the most important ; corresponding conditions may occur in the upper limb. If injury to muscles is likely to impair or destroy the mobility of a neighbouring joint, the joint must be kept in the position in which it will be of the greatest use subsequently.

2. Secondary hamorrhage.-This is almost invariably an indication that the drainage of a wound is faulty. Consequently it should not occur subsequently to operative treatment for infection, and it will practically disappear if we effect adequate mechanical drainage in the early stages. When it has occurred the case must be submitted to operation without delay, otherwise recurrence is a practical certainty. In every case where it is anatomically possible the wound should be explored and the source of bleeding found and dealt with directly by ligation of the vessel on both sides of the erosion, choosing a healthy part of the vessel wall. Faults in drainage should also be rectified at the same time. Packs and such measures should only be used as immediate temporary means of control; they lead to a false sense of security and are often ineffective in controlling the bleeding. Turpentine I regard as useless and even dangerous, as it may lead to increased sloughing in the tissues. Proximal ligation at some distance from the wound should never be adopted where direct treatment is possible; it may fail to control the bleeding primarily, and it is no safeguard against recurrence.

Subsequently to operation secondary hæmorrhage has, in my experience, been remarkably rare. It has only occurred in four cases-i.e., 0.25 per cent.-and I attribute this entirely to the methods of drainage employed. These cases are instructive as showing the cause of the hæmorrhage. In the first case, which occurred early in the series, the posterior tibial vessels yielded behind the lower end of the tibia, probably from the pressure of a drainage-tube. This is the only fatality I have had from hæmorrhage ; the patient never rallied and died in less than two hours. In the second case, at operation for extraction of shell from a deep lacerated septic wound in the middle of the thigh received some ten days before admission and in which drainage was against gravity, the femoral artery was felt pulsating in the wall of the wound. The question of ligature was considered and rejected, but the vessel gave way 36 hours later. In the third case the brachial artery was perforated some three weeks after operation by a projecting spicule of bone on the upper fragment of the humerus, which was comminuted in its upper third. The fourth case was a difficult one of comminuted fracture of the lower third of the humerus with severe infection, in which anatomical considerations prevented adequate drainage in the direction of gravity. Here the ulnar artery yielded in the antecubital fossa.
I have operated on some 15 other cases for secondary hæmorrhage, and in each there were obvious mechanical faults in the drainage.

All cases (except that first quoted, where the patient was moribund when hæmorrhage was controlled) have been treated by direct ligation on the lines indicated. In none was there any recurrence of hæmorrhage and all patients made good recoveries.

The opinions expressed in this paper are the results* of close personal observation and practice of war surgery extending now over a period of more than two years, and I believe that a more general recognition of the principles advocated, with their thorough application to all cases of infected wounds in their early stages, will help materially to improve our results and hasten the recovery of our patients. That good results can be obtained by these methods the following brief facts will, I think, bear witness:-

1. Out of all cases treated there has been only one death from sepsis. This was a very severe compound fracture of the upper end of the femur; the patient was already overcome with toxæmia when received, amputation was not practicable, and death occurred on the following day.

2. There was one death from gas gangrene, which developed rapidly in the upper half of the thigh. A high "guillotine" amputation was immediately performed, but it was impossible to get beyond the infected area.

3. Apart from three other cases of gas gangrene, in which amputation was successfully performed, only four amputations-all successful-have been done to save life. Three of these patients had been wounded some considerable time before coming under my care ; the fourth had been literally riddled, and 22 shrapnel balls had been extracted (at three sittings) before it became necessary to amputate through a knee-joint eight days after he had been wounded.

It is questionable whether some of the limbs saved were worth preserving from a functional point of view, but this does not affect the results.

4. Two cases of tetanus occurred and both patients recovered under treatment.

\section{Conolusions.}

1. Efficient mechanical drainage is the essential factor in the successful treatment of infection.

2. The cardinal principles governing drainage are: Freedom of exit ; gravity ; capillary action.

3. Drainage-tubes should not be employed unnecessarily. They should effect the purposes for which they are used, and should not act injuriously in the wound.

4. Dressings should not be tight, of close texture, or waterproof. They should be exposed to the air while discharge is profuse.

5. Oontinuous irrigation aids disinfection by mechanical removal of discharge. It is usually not feasible when dealing with large numbers of cases. It is not necessary in order to obtain good results.

6. Free administration of fluids to the patient is an important adjunct in the treatment of sepsis.

7. The value of concentrated saline solutions and antiseptics as aids to disinfection is doubtful and negligible.

8. The use of salines and antiseptics in no way diminishes the necessity for free mechanical drainage.

9. Vaccines are not helpful in dealing with infections in wounds of war.

10. Rest is an important factor in wound treatment and should never be neglected

11. Most of the complications and sequelæ of infection are due primarily to inefficient mechanical drainage, and are therefore preventable.

12. Delay in healing is almost invariably due to local mechanical causes.

My best thanks are due to Lieutenant-Colonel P. J. Freyer for the interest he has taken in my work and the kind remarks he has made on my paper.

\section{Remarks by Lieutenant-Colonel P. J. FreYer.}

Major William Pearson has invited me, as consulting surgeon to the Eastern Command, and therefore intimately associated with him in the work of the large war hospital to which he is surgically attached, to offer some observations on his paper on "Important Principles in the Drainage and Treatment of Wounds." Whilst readily complying with his request, my observations will be brief, for the simple reason that I entirely endorse the views enunciated by him, and 
that anything I might say on the subject has already been simply, clearly, and forcibly expressed in this essay.

As my mission in this war has been mainly consultative, and only to a minor degree operative, I have had ample opportunities in passing under review tens of thousands of wounds of all types and degrees and of comparing the methods and results of other surgeons, many of them amongst the ablest in the profession, and all actuated by the desire to place at the divp sal of their patients what they considered the best methods of treatment. The "supersaline" treatment with its paraphernalia was much in vogue in the early days of the war, but has been gradually disappearing from the stage. Antiseptics innumerable have, of course, been employed, and successfully employed, I have noticed, in proportion to the adequacy of the accompanying drainage. At my first visit to Major Pearson's wards, containing some 500 berls, I was much struck by the simplicity of the dressings and the thorough mechinical drainage of the wourds. Each subsequent visit confirmed me in my admiration of the surgical methods employed and the results obtained therefrom. I can honestly say that I have never seen better surgical wisk than that accomplished by the simple methods described in this $p\lrcorner$ per.

\section{THE}

INFLUENZA EPIDEMIC OF SPRING, 1915 WITH SPECIAL REFERENCE TO ANOMALOUS THROAT SIGNS.

Bi A. GEOFFreY SHerA, B.A., M B., B.C. CANTAB., M R.C.S., L.R.C.P. LOND.,

LATE LIFUTENANY, R.A.M. C. ; OFFICER IN CHARGE OF PATHOLOGICaL DEPAKTMEVT, CEVTRAL MLLTARY HOSPITAL, EASTBUURNE; LATE ClinICAL ASSISTANT, MHDICAL OUT-PATIENT DEPARTMENT, EVELINA HOSPITAL FUR GHILDREN, S.E.

\section{Introdustory.}

Thrs paper is a résumé of observations on some 500 cases of influenza in civil practice in South London, occurring about two years ago; time having hitherto been otherwise occupied, these observations conld not he sent to the press until now. At the end of 1916 we were faced with the same perennial visitor, and to demonstrate its vagaries this paper should make possible a comparison between the syndrome of spring, 1915, and that of the last months of 1916 .

It must primarily be remembered thit the influenza germ is certainly responsible for a variety of lesions, dissimilar in almost every epi lemic and in almost every locality, yet retaining certain common traits. For instance, the older physician scarcely recognises the modern type of influenza as his old friend the "knock-me-down fever," and therefore is apt to overlook it, if not to despise it as unworthy of much attention. Yet I venture to s'ate, and herein hope to show, that the clinical and bacteriological phenomena associated with this complaint are a study worthy of much attention and as yet largely unfathomed, in spite of the enormous literature on the subject.

At the onset I wish to state that although the cases of the present epidemic appear to be rather more severe, they present many of the phenomena which I am about to describe.

\section{General Description.}

Adnpting the older clissification of catarrhal, nervous, and abdominal types, one could describe the cases of the 1915 epidemic as purtaking of a combined catarrhal and nervous type. The abdominal type was almo-t completely absent. All these cases presented in the first stages a vesicular eruption on the soft palate, fauces, and pnsterior pharyngeal wall, with ervthema of the mucous membrane. and were generally followed by neuralgias or other nervous phenomena. The initial symptoms were mild, but complications were frequent and varied. The catarrh was protracted and the neronus system slow in resuming its normal tone.

To sum up, one might describe the syndrome as a febrile catarrh with a secondary neurotoxic lesion as its most prominent complication. Later statistics will be given and these lesions classified. Before going further it will be well to have a clear clinical picture.

A typical case was that of a man, aged 25, a smoker. The patient was in contact with several folk afflicted in this epidemic. At first he had a feeling of malaise, with muscular pains in the limbs, especially the thighs, also frontal head ache and disinclinatiun for work. That evening his temperature was $101^{\circ} \mathrm{F}$. and pulse 72 . The urne was febrile. H had, the day before, noted an abuodant pharyngeal catarrh, so abundant that he was continually expectorating mucas He complained of a sore throat and had some conjunctival injection.

Examination of the throat showed an erythematous patch of a dark red colour on the right side of the soft palate and uvula, which was in this case unilateral, ceasing abruptly at the middle line. Upon this erythematous patch were seen about half a dozen almost transparent vesicles, about the size of a pin's head, whose base was faintly reddened. More could be seen on that part of the soft palate where there was no erythema. The vesicles were quite firm to touch. There were vesicles also on the posterior pharyngeal wall and also some granulations. There were no adenoids or tonsils to speak of.

There was vo anterior nasal coryza. The soft palate was definitely tender to touch, and neuralgia was complained of in the teeth on the right side.

The temperature was down next morning, but there were still headache and "shivering feelings" in the back. In a day or two, the mucous discharge had ceased, but the throat was extremely sore for a week or more and the remissions lasted for a month. The general symptoms subsi ied in three days, but the vesicles recurred for three weeks or more -i.e., as long as the throat was sore. Even exposure to a cold wind would cause their reappearance after apparent subsidence. Beyond a general nervous debility the case was free from complications. After four to six weeks the hypotonia gradually wore off, with complete recovery.

Bacteriological examinations were kindly made for me by Dr. W. E. Kingdon, of Sheffield Universitv. Cultures from the vesicles were made on hæmoglobin media and stained for influenza. Only staphylococci and streptococci were found. True influenza bacilli were obtained by myself earlier from the mucous discharge on the posterior pharyngeal wall, but could only be obtained quite early in the disease.

The above somewhat, but I hope not unfuly, detailed description represents a fairly typical case. Whilst the age and sex incidence was not in any way restricted, as a general rule youth and middle-age suffered most.

\section{Analysis of Syndrome.}

Analysis of the syndrome (group of symptoms) falls under four headings, and will be illustrated :-(1) The severity of the initial coryza; (2) the erythema and persistent vesicles in the throat; (3) the sequential neuralgias; (4) the variety of complications.

1. The severity of the initial coryza.--In the above quoted case the flow of thin mucus was so severe that it was impossible for the patient to smoke at all, and he was com pelled to expectorate large quantities of mucus and saliva. At night he was kept awake by a troublesome hawking. It may be important to note that almost alwars the cough was relieved by small doses of morphia, this suggesting a nervous origin, in part.

In another case, a male aged 72 , who had had cerebral hæmorrhage about five years before, was so troubled with corvza that the strain of expectoration brought on epistaxis which could only be controlled by firm nasal tamponage.

An infant, aged 10 months, was continually salivating and coughing for three days, with much vomiting, showing the excess of mucus had been swallowed.

In another patient continual hawking and expectoration brought on a subconjunctival ecchymosis.

In a pregnant woman, aged 38, the expectoration cansed an abdominal muscular rnpture.

Such examples are sufficient to demonstrate this very unpleasant symptom.

2. The eryithema and vesicular eruption.-This complex is largely the raison d'être of this paper. In no case examined with a typncal influenza sundrome did I fail to find this clinical picture. Also it should be noted that in only one case was there an eruption (and that herpotiform, and on a mucous surface, the lips) found elsewhere on the hody.

Numbers of patients came up complaining of sore-throat, which they found difficult to get rid of, of 10 davs' duration or more, and no other symptoms save slight malsise. Examination of the soft palate, uvula, and posterior pharyngeal wall invariably showed some vesicles about the size of a pin's head, and nearly always accompanied by erythema.

Others came up complaining of facial or orbital neuralgia but no sore-throat. Examination of such cases revealed the same clinical picture; in fact, this throat syndrome one found pathognnmonic in this particu'ar epidemic of influenza.

One rather puzzling ca se of what was apparentiv influenzal neuralgia, where one expected to find vesicles and erythema, 